\title{
[ 336$]$
}

\author{
V. \\ Anelyfedes fogenanntenKonils \\ vom $M e i j \operatorname{ser}$ \\ ron
}

Prof. Stromerer in Göttingen.

(vorgelefen in der künigl. Soc. am ;. Dec. 1811.)

Diefes Folfl lindet fich bei Frankenkarn, einem am ülẗlichen Ablıange des Meijsıers gelegnen Dorle, und ift dort zuer..t vom Bergrarl Scha ub zu Allendorf, [chon ver melireren Jahren entcleckt, und fiir indentilich nit den von Retzius befchriebenen Konit gehalien worden, auch unter dielien Namen in den Mlineralienhandel gekommen. Allein lchon eine Vergleichung der äu[sern Charaktere mit denen des wahren Konits, welchen Herr Prof. Hausmar.n von feiner nordilichen Reife mitgebracht hat, liel's auf eine Verlichiedenheit beider Folfile lchliefien. Sie beftätigte fich durch die Analyle, welche den vermeintlichen Konit rom Meilsner als ein zur Formation des Bittcrkalls gehïrendes Folfil zu erkennen gab. Er enthält in roo Theilen

$$
\begin{aligned}
& \text { 32,588 Th. Magnefia } \\
& \text { 15,160 - Kalk } \\
& \text { 2,962 - Lifenoxyd }
\end{aligned}
$$




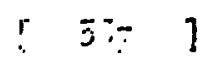

$0,5.50$ Th. Kiplelerde

4S,SUS - Kubienitiure

befteht allo in soo 'Thrilen aus

$0,25=$ - adili:irizcnde Feucluigkrit

6S,082 T!. holitenfiurer Magnefia

26,-19-kolilenlaurem Kill!e

4. 117 - kohlenfaurem Eifen

0,530 - Kielielerde

0,252 - adhitrirender Fenclutigkeit.

Dem Gefüge, dem bruch und den mehrfien übrigen phyfilch - mineralogilchen Eijenl'clatten nach, künmt er mit dem dicliten Sitteríallic von Flrublchit 2 in Mähren iaberein, welcher antangs für verhürteten Mlagnefit genonmen wurde, bis die III. Haberle und Bucholz ihn als ein daron velentlich ve:feliedenes Foliil unter der benennung dirluter Bitctrkalk unterlehiecien haben. Duch weicht er von demleliben ab durch ein grülseres lpecifilches Cowicht, welches Hr. Prof. Stromeyer bei 12,55 C. 2,972 gefunden hat, und durch eine grül'sere Härte, indern er Glas mit Leichtigkeit rizt, aun Stahle Funken-fiebt, und fich fthwer zerlprengen und nur mit Mühe pulvern lälst. Aulserdem unterlcheidet fich der Mleilsnerlche dichte Bitterkalk fowohl von dem Mährifchen, als von den ïbrigen bis jetzt bekannten und chemifch unterluchten Abänderungen diefes Folfils (als dem Bitlerfpath, dem Dolomit und dem Gurofian) hauptfïrhlich darin, dals bei ihm die knhlenliure Magnefia bedeutend vor dem kohlenlamren Kalke präponderirt, indel's bei diel'en Fof- 


\section{[ 335 ]}

filien das Limgekehehrte Statt finder.. Es frägt lich dahur, ois der Meilsner liche dichte Bitterkalk dieferiall nicht als eine befondere Abinderung der Bitterkalk-Arten aufzuliihren ili. Urbrigens erhält durch dicle Analyle die Erfahrung auls neue cine Beftitigungr, dals die liagnefia durch ilare Beinifclung im entwällierten Zuliande die Stärke des Zulaumenhangs vermehrt; und dieler Linliand verdient gewils bui Verfertigung unlerer T'ïprerwäaren mehr, als es bisher gefchehn ift, in Erwägung gezogen zu werden.

linber das geognofiifehe Vorkommen des Muilsuerichru Bir erkalks hat H.rr Prof. Stromeger fich bis rezt keine belimmte Narluricht intichatten kijnaria. Er vormuthet aber, nach den lixempliren, die itim bis jetzt zu Geficht gekommen lind, duls dielier Bitterkalk zu Frabkenhayn blols als Gelchiebe: vorknmme, indem die Stïcke alle mehr oder minder abgerunclet und iiufserlich mit Eilennxyd-Hydrat ïberzogen waren, welches lich olfenbar dureh. Zerletzung des oxydulirten kohlenliuren Eiliers gebildet hatte *).

7) Diefe Vermuluun:; ili narh drm, was ich aus Irn. BR.Scha ub's mïndiichen deufserungion vvifs, lehr gegründet. Die Griblieber linden lish, wenn ich mich niche irre, neben einoin Hohisege.

G. 